\title{
Delivery of monocyte lineage cells in a biomimetic scaffold enhances tissue repair
}

\author{
Michael S. Hu, ${ }^{1,2}$ Graham G. Walmsley, ${ }^{1,2}$ Leandra A. Barnes, ${ }^{1}$ Kipp Weiskopf, ${ }^{2}$ Robert C. Rennert, ${ }^{1}$ \\ Dominik Duscher, ${ }^{1,3}$ Michael Januszyk, ${ }^{1}$ Zeshaan N. Maan, ${ }^{1}$ Wan Xing Hong, ${ }^{1}$ \\ Alexander T.M. Cheung, ${ }^{1}$ Tripp Leavitt, ${ }^{1}$ Clement D. Marshall,' Ryan C. Ransom, ${ }^{1}$ Samir Malhotra, \\ Alessandra L. Moore, ${ }^{1}$ Jayakumar Rajadas, ${ }^{4}$ H. Peter Lorenz, ${ }^{1}$ Irving L. Weissman, ${ }^{2}$ \\ Geoffrey C. Gurtner, ${ }^{1}$ and Michael T. Longaker ${ }^{1,2}$ \\ ${ }^{1}$ Hagey Laboratory for Pediatric Regenerative Medicine, Department of Surgery, Division of Plastic and Reconstructive \\ Surgery, and 'Institute for Stem Cell Biology and Regenerative Medicine and Ludwig Center, Stanford University School \\ of Medicine, Stanford, California, USA. ${ }^{3}$ Department of Plastic and Hand Surgery, Klinikum rechts der Isar, Technical \\ University of Munich, Munich, Germany. ${ }^{4}$ Biomaterials and Advanced Drug Delivery Laboratory, Stanford University School \\ of Medicine, Palo Alto, California, USA.
}

The monocyte lineage is essential to normal wound healing. Macrophage inhibition or knockout in mice results in impaired wound healing through reduced neovascularization, granulation tissue formation, and reepithelialization. Numerous studies have either depleted macrophages or reduced their activity in the context of wound healing. Here, we demonstrate that by increasing the number of macrophages or monocytes in the wound site above physiologic levels via pullulan-collagen composite dermal hydrogel scaffold delivery, the rate of wound healing can be significantly accelerated in both wild-type and diabetic mice, with no adverse effect on the quality of repair. Macrophages transplanted onto wounds differentiate into M1 and M2 phenotypes of different proportions at various time points, ultimately increasing angiogenesis. Given that monocytes can be readily isolated from peripheral blood without in vitro manipulation, these findings hold promise for translational medicine aimed at accelerating wound healing across a broad spectrum of diseases.

Authorship note: M.S. Hu, G.G. Walmsley, and L.A. Barnes contributed equally to this work.

Conflict of interest: A patent application by M.S. Hu, G.G. Walmsley, K. Weiskopf, J. Rajadas, I.L. Weissman, G.C. Gurtner, and M.T. Longaker is pending based on the results presented in this manuscript (international application serial PCT/ US2015/041155).

License: This work is licensed under the Creative Commons Attribution 4.0 International License. To view a copy of this license, visit http:// creativecommons.org/licenses/by/4.0/

Submitted: July 13, 2017 Accepted: September 5, 2017 Published: October 5, 2017

\section{Reference information:} JCI Insight. 2017;2(19):e96260. https://doi.org/10.1172/jci. insight. 96260.

\section{Introduction}

In the United States, chronic wounds afflict an estimated 6.5 million patients and cost over $\$ 25$ billion annually (1). This social and economic burden is projected to increase with the rising incidence of diabetes, obesity, and an aging population. Consequently, there is an emergent need for wound therapies that safely and efficaciously accelerate the rate of healing. Our findings suggest that autologous macrophage or monocyte transplantation may represent a clinically viable strategy to heal chronic wounds in both nondiabetic and diabetic patients.

Cutaneous wound healing requires the activation and recruitment of many cell types, including fibroblasts, keratinocytes, endothelial, and inflammatory cells. Macrophages migrate into the wound site during the early stages of wound healing. They provide protective immune function and organizational cues for other cell types by secreting a diverse repertoire of regulatory molecules relevant to wound healing, including TGF- $\beta$ and VEGF (2). Wound macrophages are derived primarily from circulating monocytes that acquire an activated phenotype with associated surface marker changes (i.e., mannose receptor, dectin-1) upon arrival to the wound site (3).

Macrophages are thought to play a critical regulatory role in many stages of wound healing, including angiogenesis, reepithelialization, and remodeling. Evidence for the importance of macrophages in these processes comes from experiments demonstrating impaired wound healing in mice following diphtheria toxin receptor-based targeting of macrophages, genetic knockout of granulocyte/macrophage colony-stimulating factor (granulocyte/M-CSF), and administration of antimacrophage antiserum (4-6). More recently, investigators have further defined the temporal contribution of macrophages to wound healing by 
depleting macrophages during the initial inflammatory phase, the intermediate phase of granulation tissue formation, or the final remodeling phase (7). These experiments showed that macrophage depletion during the initial 4 days after injury significantly impaired the healing response, while depletion during later stages had progressively diminishing effects (7).

Although these and other studies either depleted or inhibited macrophages in the context of wound healing, few have investigated the effects of directly increasing the number of macrophages in the wound site, and mixed results have been reported $(8,9)$. Gu et al. demonstrated that injection of activated autologous monocytes/macrophages into the wound bed of full-thickness wounds promoted healing in diabetic rats (8). Jetten and colleagues showed that M2-polarized macrophages injected into full-thickness excisional wounds of wild-type and diabetic mice did not improve healing or delayed healing, respectively (9). Both studies delivered cells via injection either into the wound bed (8) or intradermally around the wound on days 1 and 3 after wounding (9). In addition, both studies utilized full-thickness excisional wounds without splinting. Rats and mice are loose-skinned mammals and that heal primarily by contraction of the panniculus carnosus, whereas humans have tight skin and heal primarily by granulation and reepithelialization (10).

Here, we investigate the effects of increasing the quantity of macrophages above physiologic levels immediately during the initial stages of cutaneous wound healing in wild-type and diabetic mice via a delivery method using a biomimetic hydrogel scaffold. Macrophages derived from the bone marrow of FVB-Tg(CAG-luc,-GFP)L2G85Chco/J mice, which express firefly luciferase and cytoplasmic EGFP constitutively in all cells (11), allowed us to track the survival and behavior of transplanted macrophages in splinted full-thickness excisional wounds using an in vivo imaging system (IVIS) and histologic analysis of GFP fluorescence. Single-cell analysis of transplanted macrophages FACS isolated from splinted cutaneous wounds during the healing process provided insights into ongoing questions regarding macrophage function in repair. To test our application in a common disease state, we also harvested macrophages from and transplanted onto FVB.BKS(D)-Lepr ${ }^{\text {db }} /$ ChuaJ mice, an animal model for type 2 diabetes mellitus. Finally, for translational application, monocytes were isolated from a healthy patient and a patient with type 2 diabetes mellitus and transplanted onto immunocompromised Crl:CD1-Foxn $1^{\text {nu }}$ mice.

\section{Results}

Macrophage transplantation accelerates wound healing. To assess the effects of directly increasing the number of macrophages during the early stages of wound healing, we isolated macrophages from the bone marrow of $F V B-T g(C A G-l u c,-G F P) L 2 G 85 C h c o / J$ mice, which express firefly luciferase and cytoplasmic EGFP constitutively in all cells (11). After 10 days in culture with M-CSF, adherent cells exhibited the characteristic morphology of macrophage differentiation as previously described (ref. 12 and Supplemental Figure 1; supplemental material available online with this article; https://doi.org/10.1172/jci.insight.96260DS1). Macrophages were then seeded (in PBS) onto pullulan-collagen composite dermal hydrogels and transplanted $\left(2.5 \times 10^{5}\right.$ cells per wound $)$ onto 6-mm full-thickness splinted excisional wounds on the dorsum of syngeneic $F V B / N J$ mice (background of donor strain matched to recipient) at the time of wounding. Mice heal wounds primarily via contraction, whereas humans heal wounds primarily by granulation and reepithelialization. By splinting the wound, contracture is minimized and mouse wounds heal with human-like kinetics via granulation and reepithelialization. At this time, control hydrogels (PBS only; no cells) were also transplanted, and wound healing outcomes were assessed. The survival, localization, and behavior of transplanted macrophages in the wound site were characterized using IVIS imaging, histologic analysis of GFP fluorescence, and gross photographic examination of wound healing progression.

Macrophage-seeded hydrogels accelerated the rate of wound healing as compared with unseeded hydrogel controls on days 4-12 ( $P<0.01$; Figure $1, \mathrm{~A}$ and $\mathrm{B})$. The average time for complete wound healing was 11.2 days in the macrophage group versus 13.4 days in the control group $(P<0.001$; Figure $1 C)$. This difference in the rate of healing was established during the first 4 days of wound healing and persisted through completion (Figure 1B). IVIS imaging showed survival of transplanted macrophages through day 7 of wound healing $(P<$ 0.05; Supplemental Figure 2A). Histologic analysis of GFP fluorescence in cryopreserved wound sections (days $1,3,5$, and 7 after wounding) confirmed survival of transplanted macrophages through day 7 of wound healing (Supplemental Figure 2B). Interestingly, the localization of transplanted macrophages gradually shifted from the upper regions of scaffold/granulation tissue to the mid and lower dermis by day 7 (Supplemental Figure 2B). We assessed CD31 (a marker for endothelial cells) staining in healed macrophage-treated wounds versus control wounds. Macrophage-treated wounds demonstrated significantly increased CD31 staining as compared with 
A

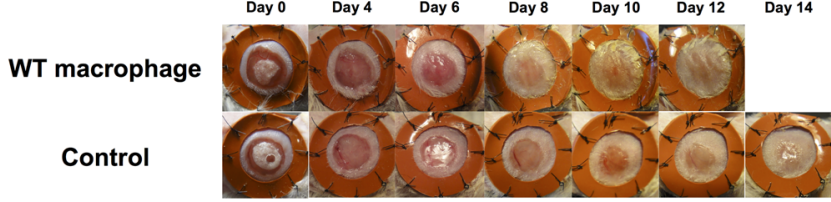

B

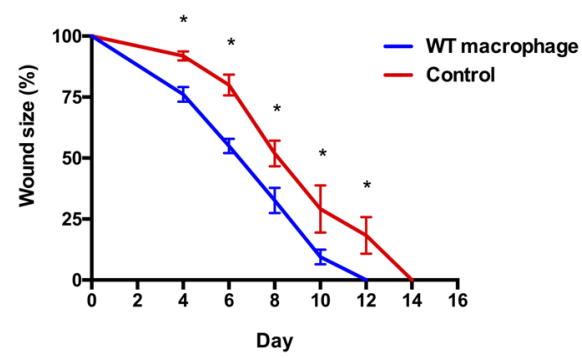

D

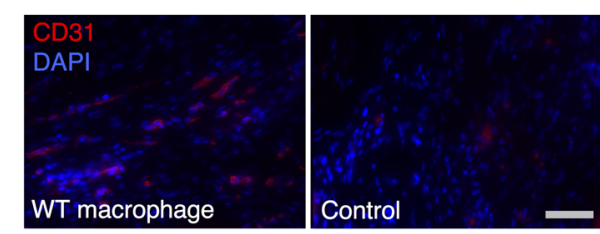

CD31 immunofluorescence

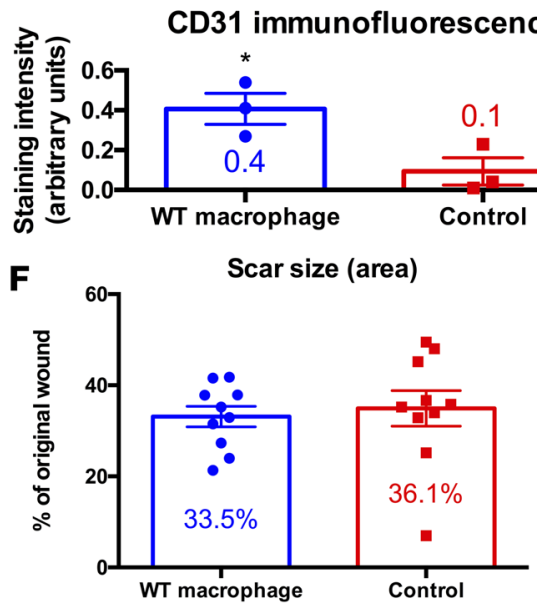

H

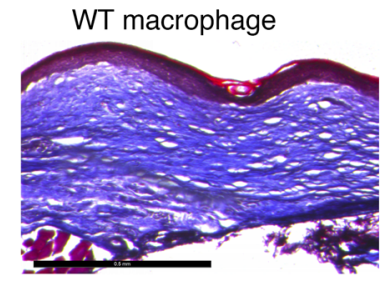

\begin{abstract}
C
\end{abstract}

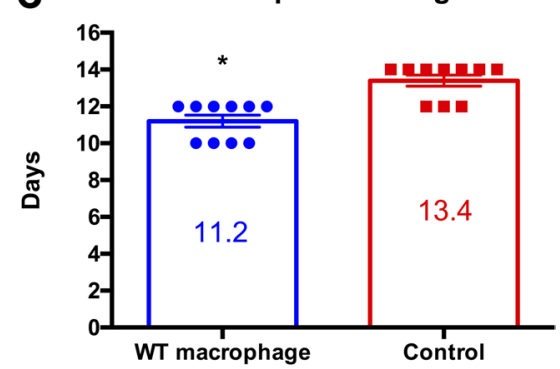

E

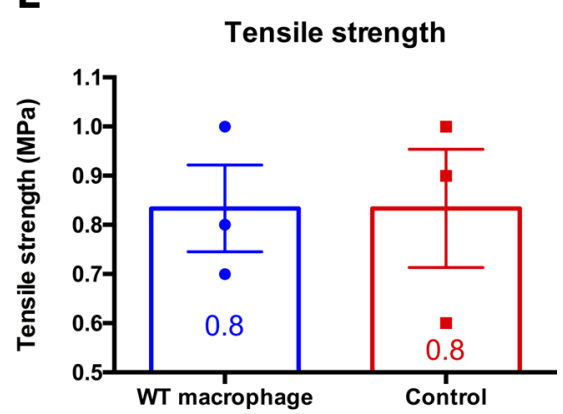

G

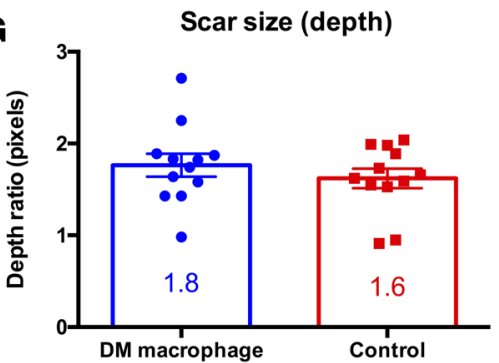

Control
Figure 1. Macrophage transplantation improves cutaneous wound repair in a humanized model of excisional wound healing in wild-type mice. (A) Gross photographs of wounds ( $n=10$ per group) during healing with macrophage-seeded hydrogel (top row) or with hydrogel control (bottom row). (B) Wound healing curve showing wound size as a percentage of original wound versus time in days ( ${ }^{*} P<0.01 ; 2$-tailed unpaired Student's $t$ test; $n=10$ per group). (C) Bar graph showing the difference in time to complete healing between macrophage-treated and control wounds in wild-type mice $\left({ }^{*} P<0.001 ; 2\right.$-tailed unpaired Student's $t$ test; $n=10$ per group). (D) CD31 immunofluorescence of fully healed wounds treated with macrophage-seeded hydrogel (top left) versus hydrogel control (top right) and bar graph with immunofluorescence quantification (bottom; ${ }^{*} P<$ 0.05; 2-tailed unpaired Student's $t$ test; $n$ = 3 per group). Scale bar: $100 \mu \mathrm{m}$. (E) Bar graph of tensile strength in fully healed wounds treated with macrophage-seeded hydrogel versus hydrogel control $(P>$

0.05; 2-tailed unpaired Student's $t$ test; $n=3$ per group). (F) Bar graph of scar size as a percentage of original wound in fully healed wounds treated with macrophage-seeded hydrogel versus hydrogel control ( $P>0.05$; 2-tailed unpaired Student's $t$ test; $n=10$ per group). (C) Bar graph of scar size as depth ratio to normal unwounded skin, as seen in histology of fully healed wounds treated with macrophage-seeded hydrogel versus hydrogel control ( $P>0.05$; 2-tailed unpaired Student's $t$ test; $n=10$ per group). (H) Masson's trichrome stain of fully healed wounds treated with macrophage-seeded hydrogel (left) versus hydrogel control (right). Scale bar: $500 \mu \mathrm{m}$.

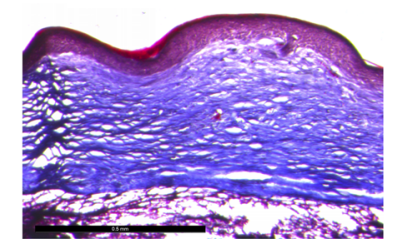

controls $(P<0.05$; Figure 1D), suggesting that macrophage transplantation leads to increased angiogenesis. This finding may explain the primary mechanism by which transplanted macrophages accelerate wound healing.

Although macrophage-treated wounds healed significantly faster than control wounds, we investigated if the acceleration of repair might have an adverse effect on the quality of healing. We first assessed tensile strength in macrophage-treated versus control wounds (Supplemental Figure 3) and found that no significant differences existed in completely healed wounds between either group (Figure 1E). Importantly, there were also no significant differences in scarring between macrophage-treated and control wounds, as determined by photographic measurement of scar area (Figure 1F) and histologic analysis of scar depth using Masson's trichrome stains (Figure 1G). Additionally, qualitative assessment of collagen density by Masson's trichrome staining revealed no discernible differences in the quality of the scar between either group (Figure $1 \mathrm{H}$ ). 
Transplanted macrophages differentiate into a mixed M1/M2 phenotype during wound healing. In order to understand the mechanism by which macrophages delivered to wounds accelerated healing, we performed a single-cell analysis of cultured cells (day 0 ) and those FACS isolated from wounds after scaffold seeding and transplantation 1, 4, and 7 days after application (Figure 2A) as previously described (13). Transcriptional profiles were simultaneously evaluated for more than 90 gene targets across approximately 70 individual cells per time point, specifically looking at genes relating to cell proliferation, inflammatory pathways, and macrophage phenotype (Supplemental Table 1).

Gene expression data were clustered according to profiles for M1, M2a, M2b, M2c, and M2d subpopulations of macrophages (Figure $2 \mathrm{~B}$ ). These data reveal a shift in the expression profile over the course of transplantation (Figure 2C). After culture and prior to placement onto the scaffold and onto the wound, the majority (73\%) of macrophages expressed an M2a phenotype, with the remaining macrophages $(27 \%)$ expressing genes characterized by M1 macrophages. After 1 day in the wound, there was a large presence of M2b (42\%) and M2c (25\%) macrophages, with a sudden decrease in the M2a phenotype (12\%). Interestingly, by day 4 , the majority of macrophages expressed the M2a phenotype (66\%), which persisted until day 7 (42\%). The M2d macrophages were first seen on day 4 after transplantation (1\%) and had a presence by day 7 (16\%). These fluctuating gene expression profiles allude to the complex well-orchestrated mechanism of wound healing, but the functional significance is not fully understood.

Transplanted macrophages express acute-phase reactants. In addition, data from single-cell gene expression analysis were depicted to compare cultured macrophages and macrophages seeded onto pullulan-collagen composite dermal hydrogel and placed onto full-thickness excisional wounds for 24 hours. Confirming the fidelity of the assay and purity of the cells analyzed, hematopoietic lineage ( $C d 45)$ and general macrophage marker (Emr1, Cd64, Itgam) genes were expressed in both cultured macrophages and those isolated from wounds, although heterogeneity was observed (Figure 3A). Kolmogorov-Smirnov analysis of these data identified multiple genes that were differentially expressed across conditions. Specifically, the expression of acutephase reactants ( $C c l 2, C c l 3$, Tnfa) was significantly upregulated upon exposure to the wound (Figure 3B).

Consistent with previous reports of wound macrophages demonstrating a complex phenotype (7) and Figure 2, macrophages in this assay displayed activation of both classical (M1: Tnfa, Cd14, Ilo) and alternative (M2: Cd86, Mrc1, Clec10a, Cd163, Mmp9, Il4r, Klf4) markers after being in the wound environment for 24 hours, although there was a predominant M2 phenotype in this setting (Figure $2 \mathrm{C}$ and Figure $3 \mathrm{~B}$ ). Consistent with the luciferase tracking data demonstrating a limited cell presence at 7 days after injury, macrophages downregulated proliferation-related genes (Pcna, Ctnnb1, Ccnd1) once in the wound environment (Supplemental Figure 4). Together, these data suggest that macrophages delivered at the time of injury accelerate wound healing by transiently "jump-starting” the inflammatory and neovascular signaling cascades. However, the functional significance of this gene expression data has yet to be elucidated.

Macrophage transplantation accelerates wound healing in diabetic mice. Given that macrophages lead to increased rates of wound healing in syngeneic wild-type mice, we next assessed the effect of transplanting macrophages from wild-type [FVB-Tg(CAG-luc,-GFP)L2G85Chco/J] mice into diabetic [FVB.BKS(D)-Lepr ${ }^{\text {db }} / C h u a J$ mice. These leptin receptor-deficient mice have long-term hyperglycemia, primarily due to severe insulin resistance that persists in the fed state, despite continued extreme hyperinsulinemia, and have been used as a model of obesity diabetes (14). Consistent with data from wild-type recipients, diabetic mice treated with macrophages from wild-type mice demonstrated an increased rate of wound healing $(P<0.05$; Supplemental Figure 5, A and B). As diabetic mice heal wounds slower than their wild-type counterparts, the control unseeded hydrogel group demonstrated a mean healing time of 20.3 days versus 17.2 days for the macrophage-treated group $(P<0.01$; Supplemental Figure 5C). There was no difference in scar size by gross macroscopic visualization (Supplemental Figure 5D) or scar depth via histology (Supplemental Figure 5E). Interestingly, the macrophages survived in the diabetic wound through complete healing $(P<0.05$; Supplemental Figure $5 \mathrm{~F})$.

Given the ability of macrophages from wild-type mice to enhance repair in diabetic mice, we sought to explore whether macrophages from diabetic mice could reproduce this effect. Macrophages were harvested from diabetic $\left[F V B . B K S(D)-L e p r^{d b} / C h u a J\right]$ mice and transplanted onto wild-type $(F V B / N J)$ mice and mice of the same strain [FVB.BKS(D)-Lepr ${ }^{\text {db }} /$ ChuaJ], as described above.

When hydrogels seeded with macrophages from diabetic mice were transplanted onto wild-type mice, wound healing was accelerated compared with unseeded hydrogel controls on days 4-12 in diabetic mice $(P<0.05$; Supplemental Figure 6, A and B). The average time for complete wound healing was 11.6 days in the macrophage-treated group versus 13.2 days in the control group $(P<0.01$; Supplemental Figure $6 \mathrm{C})$. 
A
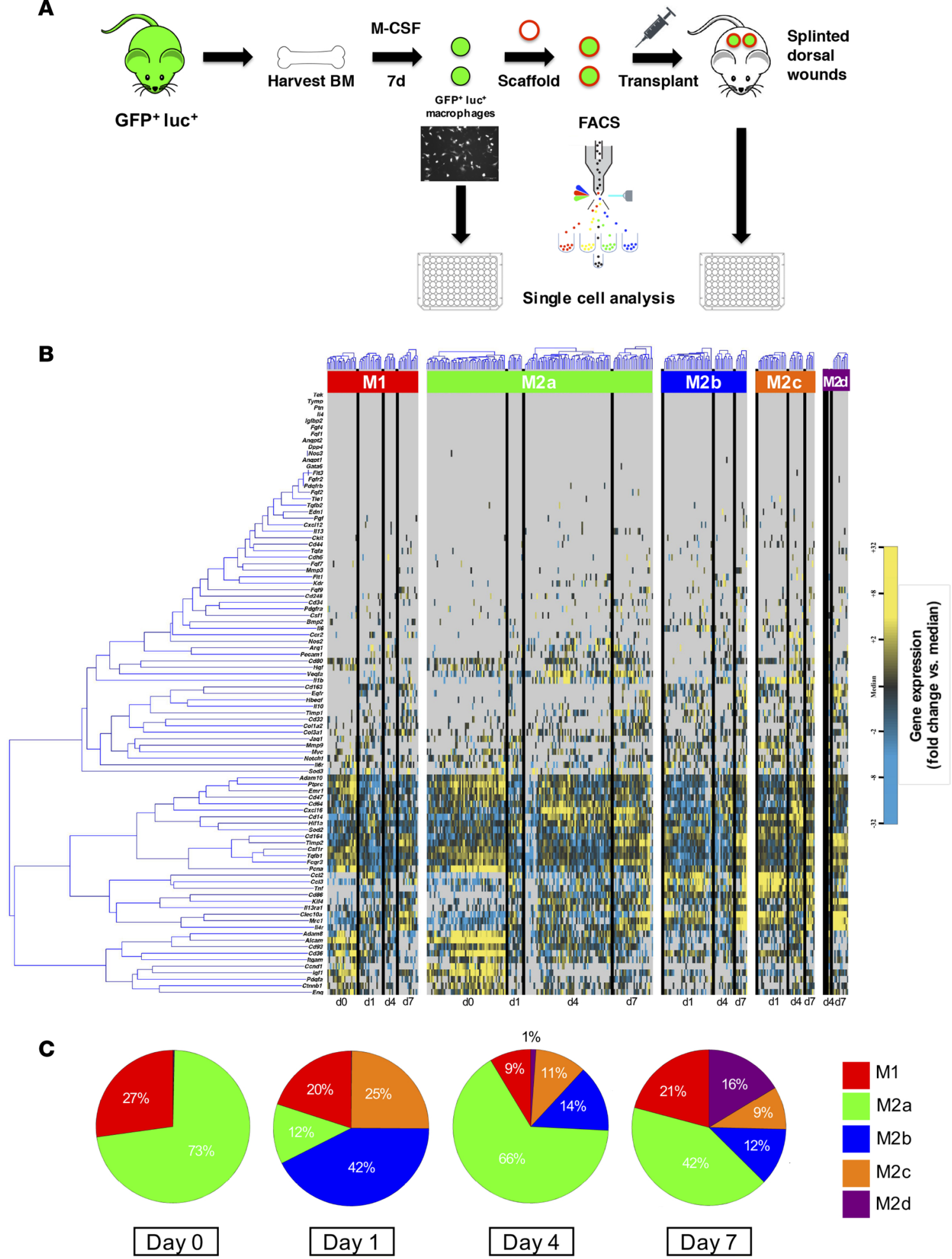

Day 4

Day 7

Figure 2. Macrophage differentiation following transplantation. (A) Schematic detailing the macrophage harvest, transplantation, and FACS isolation. (B) Hierarchical clustering of macrophages from day 0 (directly from culture) and days 1, 4, and 7 after seeding onto hydrogel and transplantation onto wounds based on macrophage phenotype (M1, M2a, M2b, M2c, and M2d). Gene expression is presented as fold change from median on a color scale from yellow (high expression, 32-fold above median) to blue (low expression, 32-fold below median), with gray indicating no expression. (C) Pie chart of macrophage phenotypes, as determined by hierarchical clustering of cells from day 0 (directly from culture) and days 1,4 , and 7 after seeding onto hydrogel and transplantation onto wounds. 
A

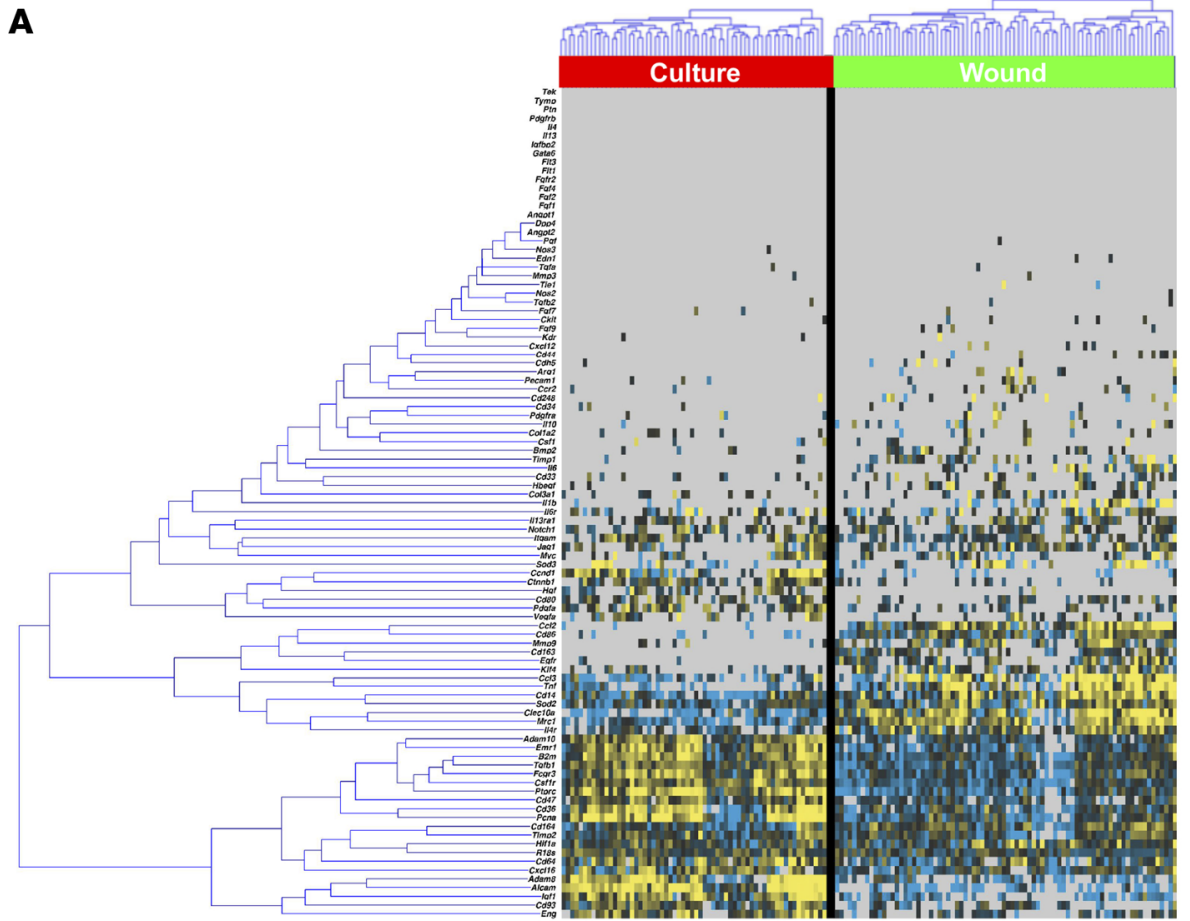

B
Acute phase reactants:

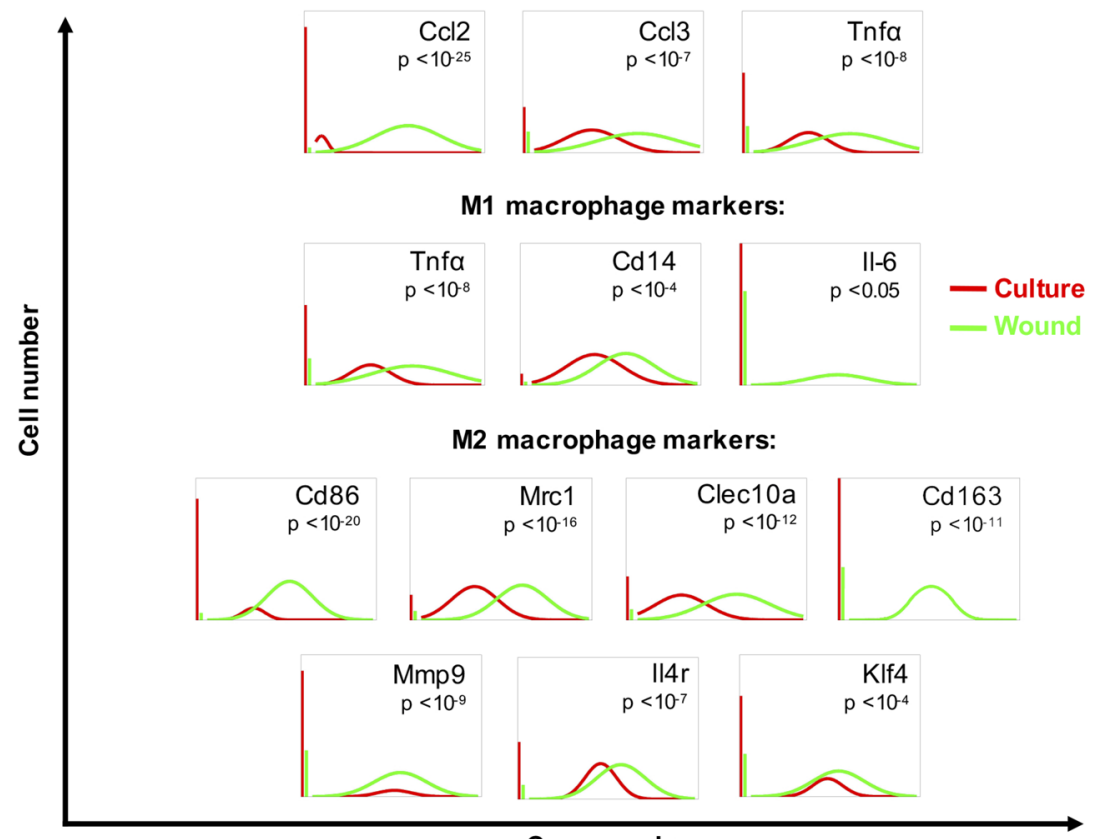

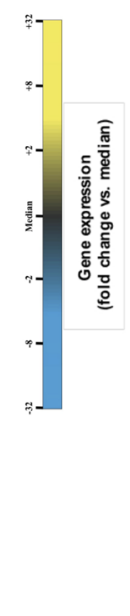

Copy number
Figure 3. Transcriptional response of transplanted macrophages.

(A) Hierarchical clustering of cells from culture (left; red) and 24 hours after seeding onto hydrogel and placement onto wound (right; green). Gene expression is presented as fold change from median on a color scale from yellow (high expression, 32-fold above median) to blue (low expression, 32-fold below median), with gray indicating no expression. See Supplemental Figure 4 for the complete data set. (B) Differentially expressed genes between culture and wound macrophages identified using nonparametric two sample Kolmogorov-Smirnov testing. Thirty-seven genes exhibited significantly different $(P<0.05)$ distributions of single-cell expression between populations, with selected acute-phase reactants and M1/ M2 markers illustrated here using median-centered Gaussian curve fits. The left vertical bar for each panel represents the fraction of qPCR reactions that failed to amplify in each group.

Although macrophage-treated wounds healed significantly faster than control wounds, there were no significant differences in scarring, as seen by photographic measurement of scar area (Supplemental Figure 6D) and histologic analysis of scar depth (Supplemental Figure 6E).

Given, the ability of macrophages from wild-type mice to enhance repair in diabetic mice, we transplanted macrophages from diabetic mice onto diabetic mice. This would have tremendous translational implications for autotransplantation of macrophages in diabetic patients with nonhealing wounds. Hydrogels seeded with macrophages from diabetic mice accelerated the rate of wound healing in diabetic mice as compared with unseeded hydrogel controls on days 4-20 ( $P<0.05$; Figure 4 , A and B). The average time for complete wound healing was 17.5 days in the diabetic macrophage-treated group versus 20.3 days in 
A

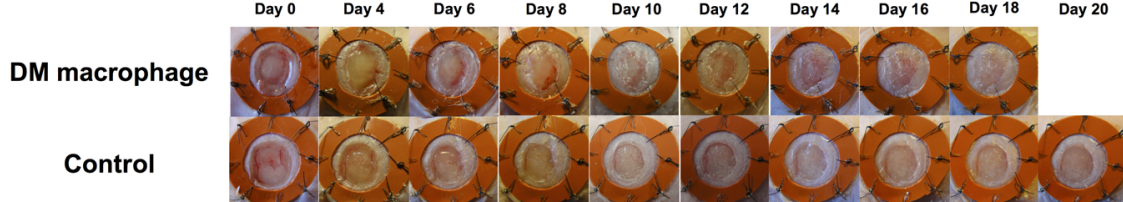

B

Wound healing curve

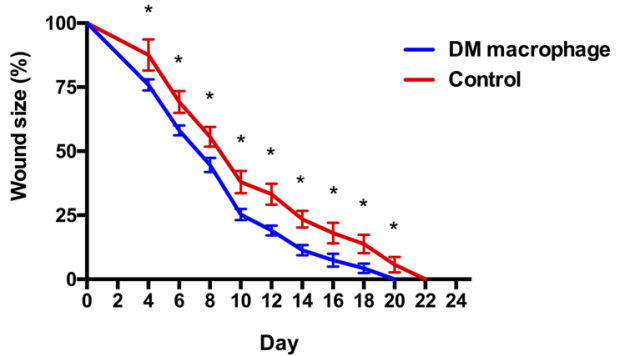

D
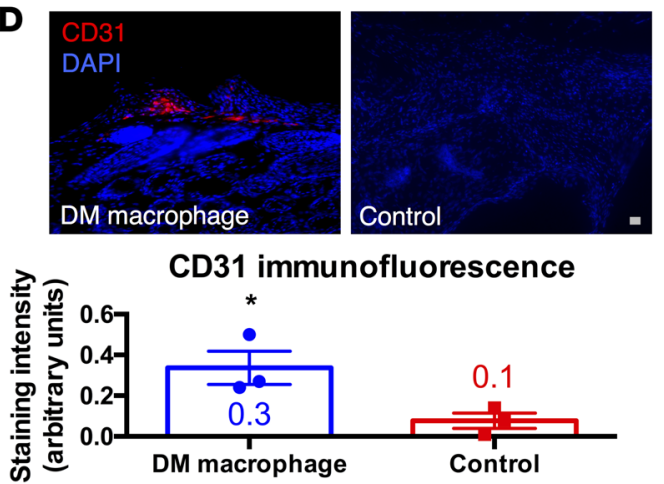

$\mathbf{F}$

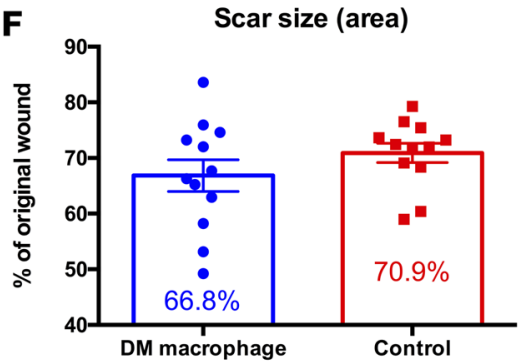

H DM macrophage

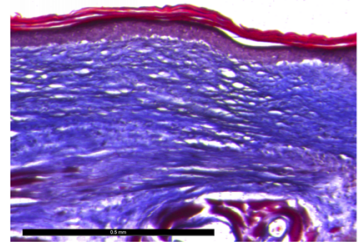

C

Complete healing

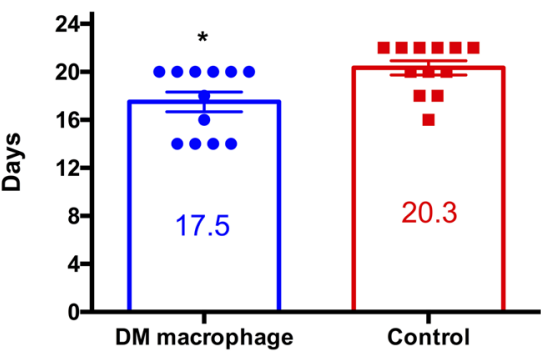

E

Tensile strength
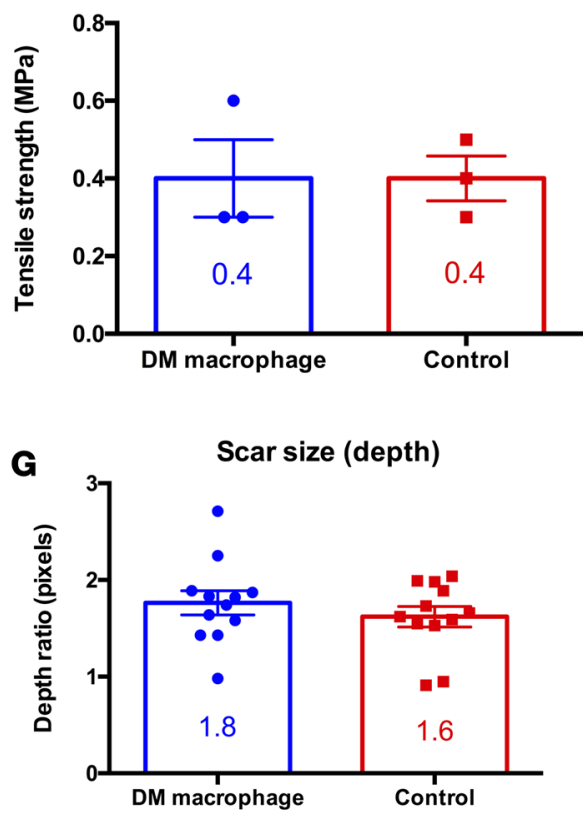

Control

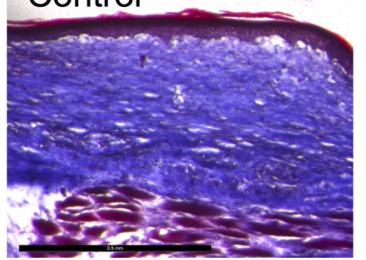

Figure 4. Macrophage transplantation from diabetic mice improves repair of full-thickness excisional wounds in diabetic mice. (A) Gross photographs of wounds ( $n=12$ per group) during healing with diabetic macrophage-seeded hydrogel (top row) or with hydrogel control (bottom row). (B) Wound healing curve showing wound size as a percentage of original wound versus time in days ( ${ }^{*} P<0.05 ; 2$-tailed unpaired Student's $t$ test; $n=12$ per group). (C) Bar graph showing the difference in time to complete healing between diabetic macrophage-treated and control wounds in diabetic mice ${ }^{*} P<0.05$; 2-tailed unpaired Student's $t$ test; $n$ $=12$ per group). (D) CD31 immunofluorescence of fully healed wounds in diabetic mice treated with diabetic macrophage-seeded hydrogel (top left) versus hydrogel control (top right) and bar graph with immunofluorescence quantification (bottom; * $P$ $<0.05$; 2-tailed unpaired Student's $t$ test; $n=3$ per group). Scale bar: $100 \mu \mathrm{m}$. (E) Bar graph of tensile strength in fully healed wounds in diabetic mice treated with diabetic macrophage-seeded hydrogel versus hydrogel control $(P>0.05$; 2-tailed unpaired Student's $t$ test; $n=3$ per group). (F) Bar graph of scar size as a percentage of original wound in fully healed wounds in diabetic mice treated with diabetic macrophage-seeded hydrogel versus hydrogel control $(P$ $>0.05$; 2-tailed unpaired Student's $t$ test; $n=12$ per group). (C) Bar graph of scar size as depth ratio to normal unwounded skin, as seen in histology of fully healed wounds in diabetic mice treated with diabetic macrophage-seeded hydrogel versus hydrogel control $(P>0.05$; 2-tailed unpaired Student's $t$ test; $n=12$ per group). (H) Masson's trichrome stain of fully healed wounds in diabetic mice treated with diabetic macrophage-seeded hydrogel versus hydrogel control. Scale bar: $500 \mu \mathrm{m}$.

the control group $(P<0.05$; Figure $4 C)$. Quantitative histologic analysis of diabetic macrophage-treated wounds demonstrated significantly increased CD31 staining, as compared with control wounds $(P<0.05$; Figure 4D), suggesting that transplanted diabetic macrophages lead to increased angiogenesis in diabetic mice. As with our prior wild-type wound studies, treatment with diabetic macrophages did not significantly affect tensile strength of fully healed diabetic wounds (Figure 4E). Interestingly, tensile strength of healed wounds in diabetic mice was less than that of wild-type mice (Figure 1E). Although macrophage-treated wounds healed significantly faster than control wounds, there were no significant differences in scarring, as seen by photographic measurement of scar area (Figure $4 \mathrm{~F}$ ) and histologic analysis of scar depth using Masson's trichrome stains (Figure 4G). Qualitative assessment of collagen density showed no significant differences by Masson's trichrome staining (Figure 4H). 
A
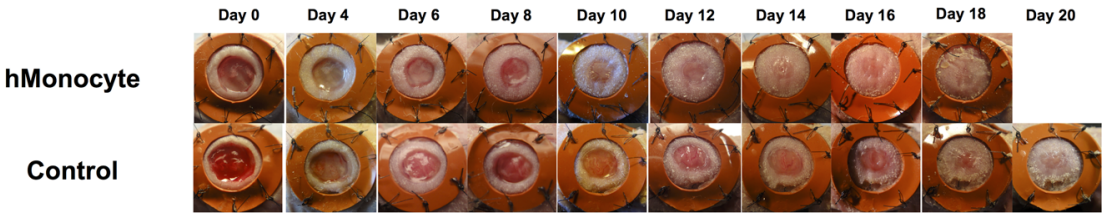

B Wound healing curve

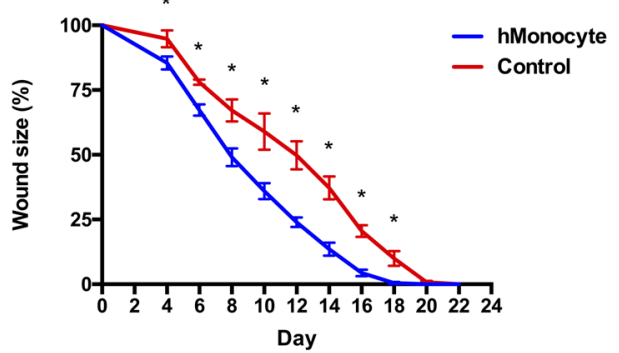

D

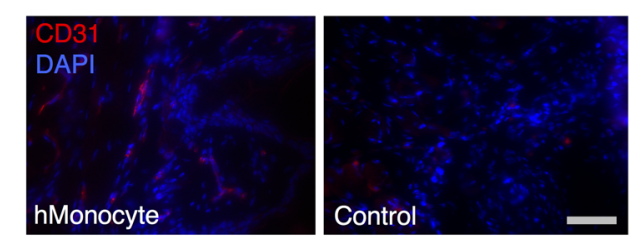

CD31 immunofluorescence

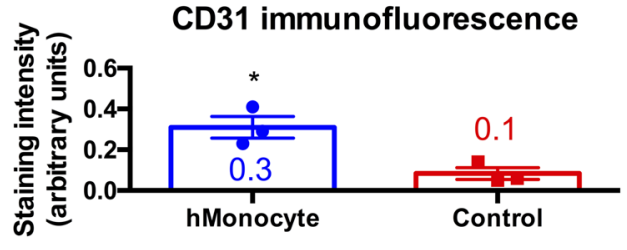

F Scar size (area)

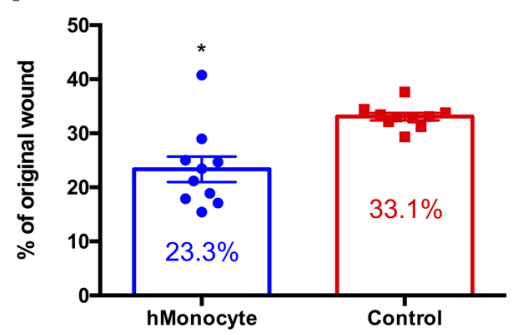

H hMonocyte

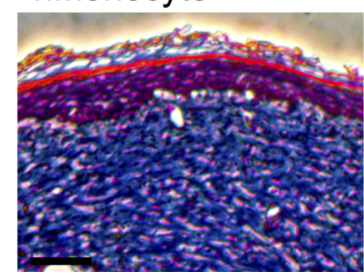

C

Complete healing

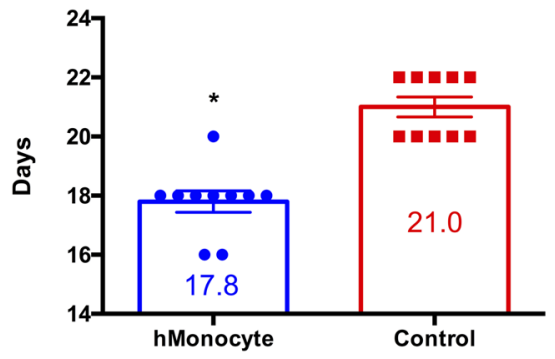

E
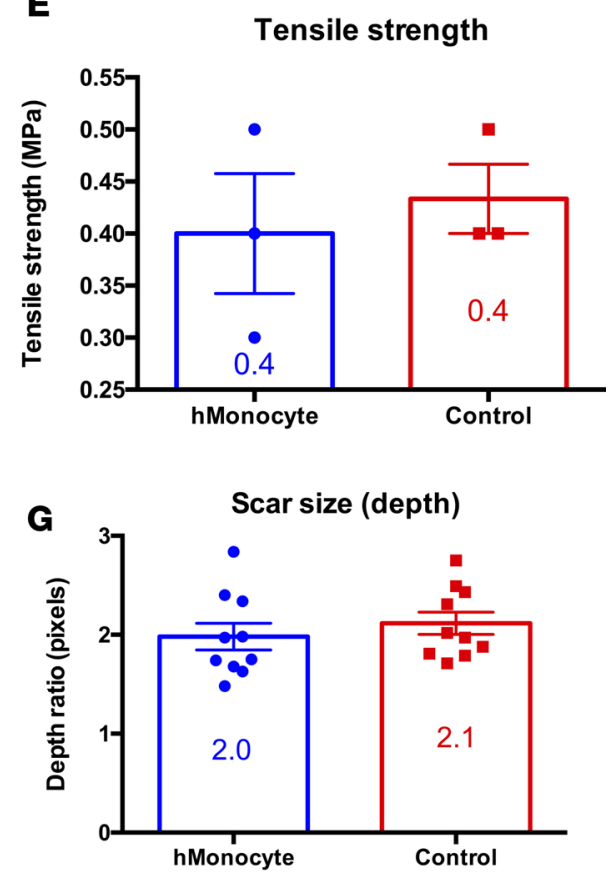

Control

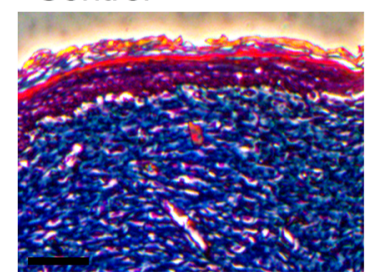

Figure 5. Human monocyte transplantation improves cutaneous wound repair in immunocompromised mice. (A) Gross photographs of wounds ( $n=10$ per group) during healing with human monocyte-seeded hydrogel (top row) or with hydrogel control (bottom row). (B) Wound healing curve showing wound size as a percentage of original wound versus time in days $\left({ }^{*} P<0.05 ; 2\right.$-tailed unpaired Student's $t$ test; $n=10$ per group). (C) Bar graph showing the difference in time to complete healing between human monocyte-treated and control wounds in immunocompromised mice $\left({ }^{*} P<0.0001 ; 2\right.$-tailed unpaired Student's $t$ test; $n=10$ per group). (D) CD31 immunofluorescence of fully healed wounds treated with human monocyte-seeded hydrogel (top left) versus hydrogel control (top right), and bar graph with immunofluorescence quantification (bottom; ${ }^{*} P<0.05$; 2 -tailed unpaired Student's $t$ test; $n=3$ per group). Scale bar: $100 \mu \mathrm{m}$. (E) Bar graph of tensile strength in fully healed wounds treated with human monocyte-seeded hydrogel versus hydrogel control $(P>0.05 ; 2$-tailed unpaired Student's $t$ test; $n=3$ per group). (F) Bar graph of scar size as a percentage of original wound in fully healed wounds treated with human monocyte-seeded hydrogel versus hydrogel control ( ${ }^{*} P<0.001$; 2-tailed unpaired Student's $t$ test; $n=10$ per group). (G) Bar graph of scar size as depth ratio to normal unwounded skin, as seen in histology of fully healed wounds treated with human monocyte-seeded hydrogel versus hydrogel control $(P$ $>0.05$; 2-tailed unpaired Student's $t$ test; $n=10$ per group). (H) Masson's trichrome stain of fully healed wounds treated with human monocyte-seeded hydrogel versus hydrogel control. Scale bar: $100 \mu \mathrm{m}$.

Human monocyte transplantation accelerates wound healing in immunocompromised mice. As transplanted macrophages were able to differentiate and promote wound healing, recapitulation of these results with blood-derived monocytes would have a tremendous translational potential, precluding the need for in vitro culture and allowing direct transplantation following phlebotomy. To assess the effects of directly increasing the number of monocytes on wound healing, we isolated human monocytes from healthy and diabetic blood donors by CD14 magnetic activated cell sorting (MACS), a potentially clinically viable isolation strategy. Human monocytes were then seeded (in PBS) onto pullulan-collagen composite dermal hydrogels and transplanted $\left(2.5 \times 10^{5}\right.$ cells per wound) onto 6-mm full-thickness splinted excisional wounds on the dorsum of immunodeficient $C r l: C D 1-F o x n 1^{n u}$ mice at the time of wounding. At this time, control unseeded hydrogels were similarly transplanted, and wound healing outcomes were assessed. 
Human monocyte-seeded hydrogels from healthy patients accelerated the rate of wound healing as compared with unseeded hydrogel controls on days $4-18(P<0.05$; Figure $5, \mathrm{~A}$ and $\mathrm{B})$. The average time for complete wound healing was 17.8 days in the monocyte group versus 21 days in the control group $(P<0.0001$; Figure 5C). This difference was established during the first 4 days of wound healing and persisted through completion (Figure $5 B$ ). Interestingly, immunodeficient nude mice heal wounds slower than wild-type mice with healing kinetics resembling diabetic mice. Given that mouse macrophage-treated wounds demonstrated increased CD31 staining as compared with control wounds, we next assessed CD31 expression in human monocyte-treated and control wounds. Monocyte-treated wounds demonstrated significantly increased CD31 staining as compared with control wounds $(P<0.05$; Figure $5 \mathrm{D})$, suggesting that monocytes lead to increased angiogenesis. This finding may explain the mechanism by which transplanted human monocytes, like macrophages, accelerate wound healing. Tensile strength testing revealed no significant differences in the strength of healed skin in monocyte-treated wounds versus control wounds (Figure 5E). Human monocyte-treated wounds healed significantly faster than control wounds, with significantly diminished scarring by photographic measurement of the scar area $(P<0.001$; Figure 5F). Scar depth was not significantly affected, as measured by Masson's trichrome staining (Figure 5G), and qualitative assessment of collagen density revealed no discernible differences by Masson's trichrome staining (Figure 5H).

Given enhanced repair with transplantation of human monocytes, we sought to determine whether human monocytes from a diabetic patient would also accelerate repair. This would further translate our findings for autotransplantation of blood-derived monocytes as a therapeutic for intractable wounds in diabetic patients. Human monocyte-seeded pullulan-collagen composite dermal hydrogels from diabetic patients accelerated the rate of wound healing as compared with unseeded hydrogel controls on days 4-20 $(P<0.05$; Figure 6, A and B). The average time for complete wound healing was 18.2 days in the diabetic human monocyte group versus 21.2 days in the control group $(P<0.0001$; Figure 6C). Similar to when human monocytes from a healthy patient were transplanted, the difference with transplantation of diabetic human monocytes was established during the first 4 days of healing and persisted through completion (Figure 6B). Like in previous experiments, diabetic human monocyte-treated wounds demonstrated significantly increased CD31 staining as compared with control wounds $(P<0.05$; Figure $6 \mathrm{D})$, suggesting that monocytes lead to increased angiogenesis. Tensile strength testing revealed no significant differences in the strength of healed skin in monocyte-treated wounds versus control wounds (Figure 6E). Diabetic human monocyte-treated wounds healed significantly faster than control wounds, with significantly diminished scarring by photographic measurement of scar area $(P<0.05$; Figure $6 \mathrm{~F})$. Scar depth was not significantly affected, as measured by Masson's trichrome staining (Figure 6G), and qualitative assessment of collagen density revealed no significant differences by Masson's trichrome staining (Figure 6H).

\section{Discussion}

Macrophages are essential for successful wound healing. Wound macrophages promote many aspects of repair, including dissolving fibrin, phagocytosing dead tissue, stimulating angiogenesis, promoting granulation tissue formation, secreting extracellular matrix, and reepithelializing the wound. After wounding, monocytes migrate into the wound through blood vessel walls and mature into macrophages. However, the number of monocytes in the wound site does not peak until 1.5 days after cutaneous injury (15). Given the role of macrophages in initiating and orchestrating numerous aspects of wound healing, transplanting macrophages directly into the wound site immediately upon wounding may effectively "jump-start" the sequence of events necessary for successful wound healing. This "jump-start" effect coupled with the persistence of supraphysiologic levels of macrophages through the initial days of healing may be responsible for the observed increase in the rate of wound repair.

Macrophages recruited to wound sites are often described as alternatively activated, requiring induction by either IL4 or IL13, and are characterized by expression of arginase-1, dectin-1, mannose receptor, and $\mathrm{Ym} 1$ (3). The alternatively activated phenotype is associated with the regulation of humoral immunity as well as allergic and antiparasitic responses (16). Single-cell analysis of transplanted macrophages isolated during wound healing provided clues as to the mechanisms by which transplantation of macrophages enhances wound healing. Transplanted macrophages in the wound site rapidly upregulated several alternative markers by day 1 after wounding. This finding is consistent with the observation that alternatively activated wound macrophages are associated with a higher degree of vascularization (16). Together, these 


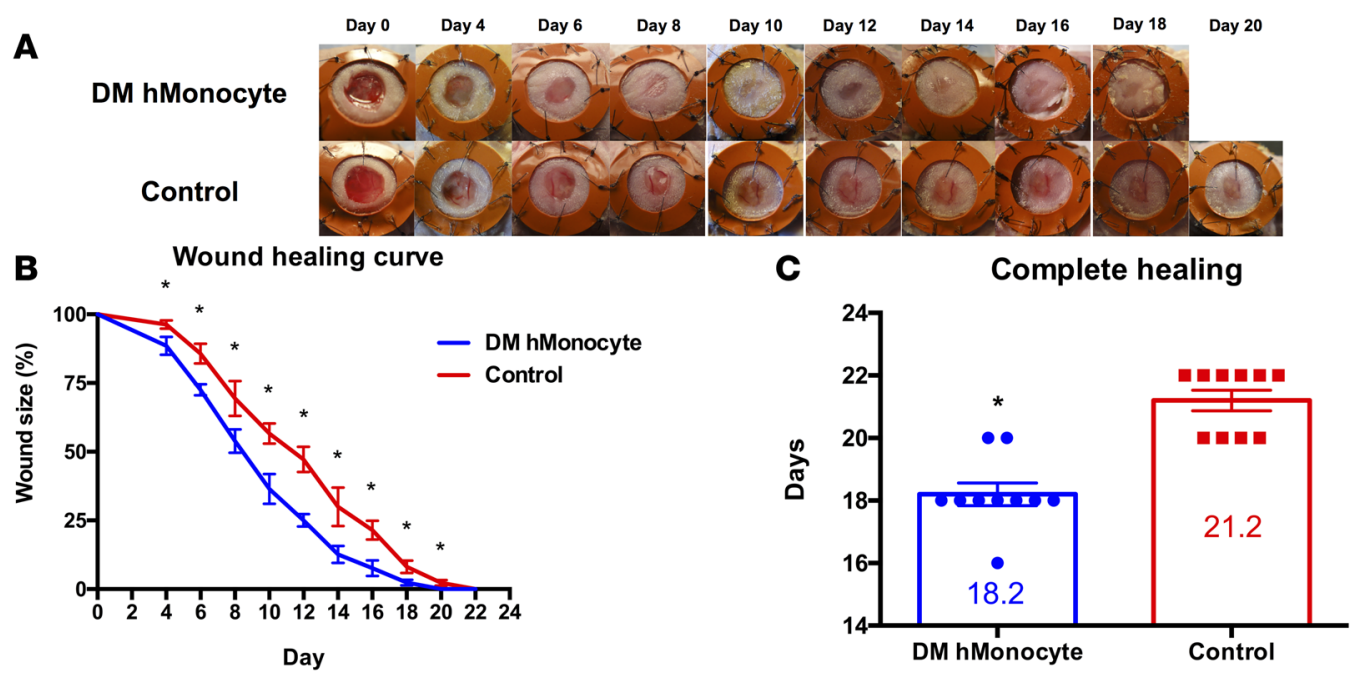

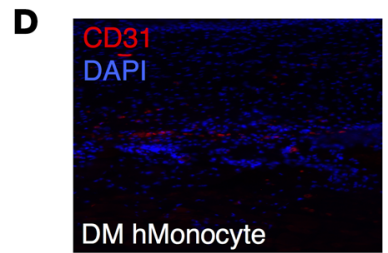
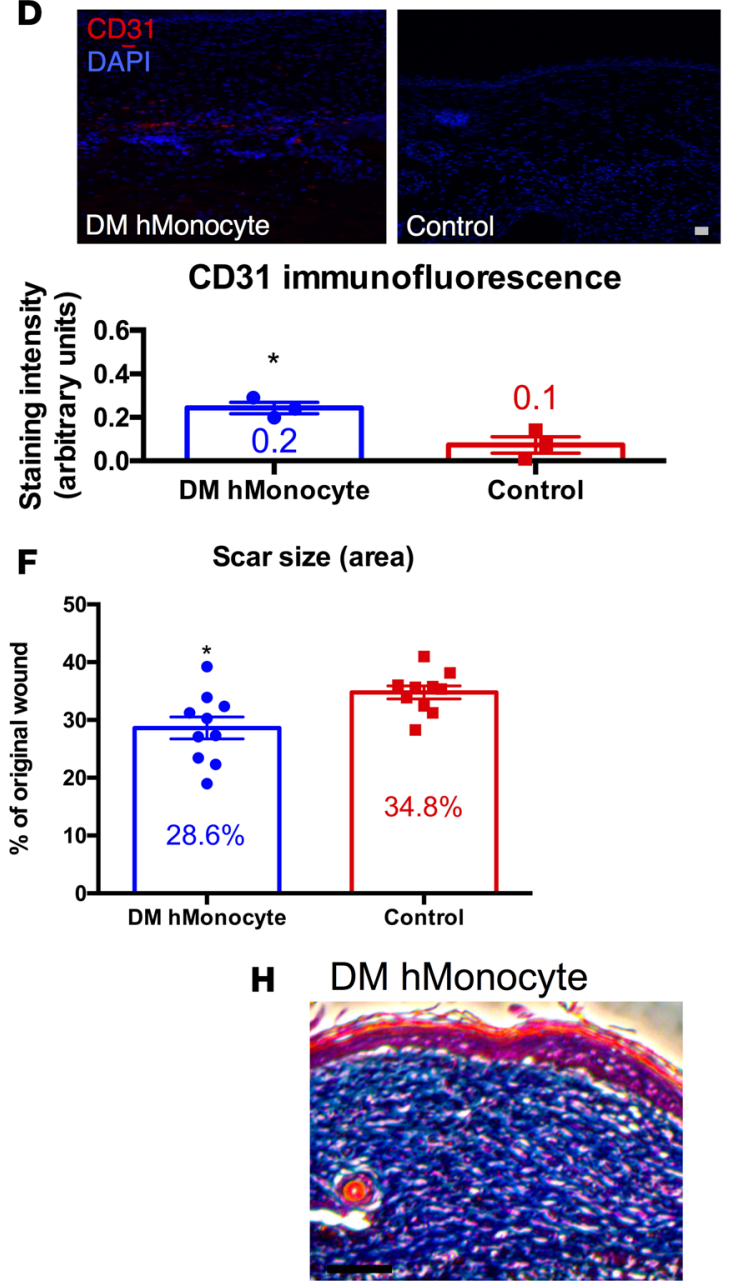

E

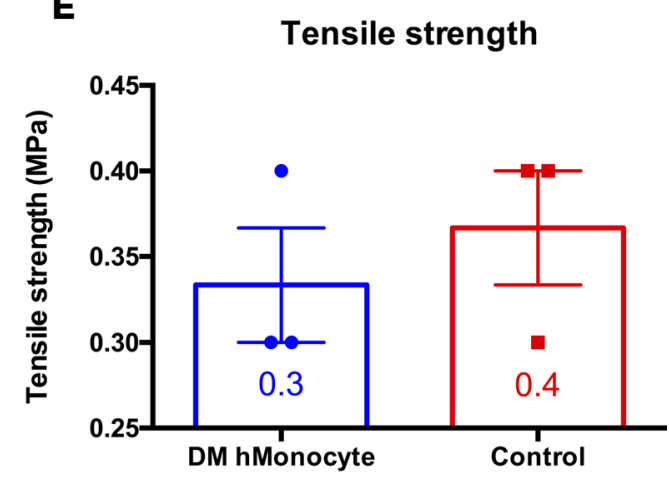

G

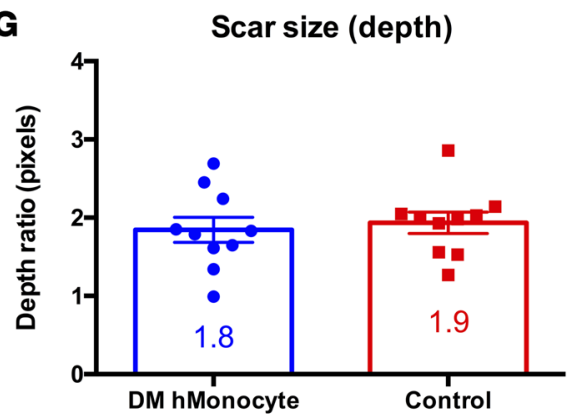

Control

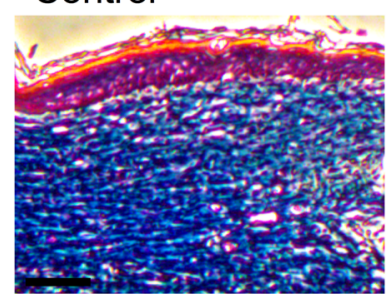

Figure 6. Human monocytes from diabetic patients improves wound repair in immunocompromised mice. (A) Gross photographs of wounds $(n=10$ per group) during healing with diabetic human monocyte-seeded hydrogel (top row) or with hydrogel control (bottom row). (B) Wound healing curve showing wound size as a percentage of original wound versus time in days ( ${ }^{*} P<0.05 ; 2$-tailed unpaired Student's $t$ test; $n=10$ per group). (C) Bar graph showing the difference in time to complete healing between diabetic human monocyte-treated and control wounds in immunocompromised mice $\left(^{*} P<0.0001\right.$; 2-tailed unpaired Student's $t$ test; $n=10$ per group). (D) CD31 immunofluorescence of fully healed wounds treated with diabetic human monocyte-seeded hydrogel (top left) versus hydrogel control (top right), and bar graph with immunofluorescence quantification (bottom; ${ }^{*} P<0.05$; 2-tailed unpaired Student's $t$ test; $n=3$ per group). Scale bar: $100 \mu \mathrm{m}$. (E) Bar graph of tensile strength in fully healed wounds treated with diabetic human monocyte-seeded hydrogel versus hydrogel control ( $P>0.05 ; 2$-tailed unpaired Student's $t$ test; $n=3$ per group). (F) Bar graph of scar size as a percentage of original wound in fully healed wounds treated with diabetic human monocyte-seeded hydrogel versus hydrogel control $\left({ }^{*} P<0.05 ; 2\right.$-tailed unpaired Student's $t$ test; $n=$ 
10 per group). (G) Bar graph of scar size as depth ratio to normal unwounded skin, as seen in histology of fully healed wounds treated with diabetic human monocyte-seeded hydrogel versus hydrogel control ( $P>0.05$; 2-tailed unpaired Student's $t$ test; $n=10$ per group). (H) Masson's trichrome stain of fully healed wounds treated with diabetic human monocyte-seeded hydrogel versus hydrogel control. Scale bar: $100 \mu \mathrm{m}$.

data suggest that macrophages delivered at the time of injury accelerate wound healing by transiently "jump-starting" the inflammatory and neovascular signaling cascades.

For our experiments, macrophages were differentiated in vitro using M-CSF, which is believed to be the major factor promoting macrophage differentiation under normal physiological conditions. M-CSF has also been reported to polarize macrophages in tumors toward an alternatively activated "M2" phenotype (17). Macrophages in this state participate in wound healing by several mechanisms, including secretion of VEGF to increase angiogenesis. Given the increase in the expression of several M2 phenotype genes in macrophages FACS isolated from wounds 24 hours after application as compared with cultured macrophages (Figure 3 and Supplemental Figure 4), it is clear that macrophage plasticity allows for further upregulation of M2 and angiogenic genes after transplantation into the wound microenvironment. Additionally, this observation implies that the macrophage phenotype is not binary and varying degrees of M2 activation exist in vivo in a wound.

We showed that transplantation of macrophages will accelerate healing in both wild-type and diabetic mice whether the macrophages are derived from wild-type or diabetic mice. This holds great promise for clinical autotransplantation. Patients most often suffering from intractable wounds have comorbidities such as diabetes mellitus. Furthermore, results from our human monocyte studies corroborate these findings. When human monocytes derived from healthy or diabetic individuals were transplanted into immunocompromised nude mice, wound repair was significantly accelerated. Interestingly, diabetic and healthy human monocytes decreased gross scar size, whereas diabetic and wild-type mouse macrophages did not. These findings portend a clinical cell-based therapy to accelerate wound repair with several advantages (Figure 7): human monocytes can be rapidly and noninvasively isolated from peripheral blood via cell sorting, transplantation can occur without cell culture directly onto a biomimetic scaffold, and outcome is not affected by diabetes.

Contrary to other macrophage delivery studies, we performed all experiments on splinted cutaneous wounds. The murine splinted full-thickness excisional wound model is accurate and reproducible, minimizes wound contraction, and allows wound healing to occur through the processes of granulation and reepithelialization, approximating human wound repair (10). As mice have evolved to rapidly heal wounds, our data demonstrating acceleration of wound healing by approximately $15 \%$ are promising for translation to humans. Additionally, we delivered our macrophages using a biomimetic pullalan-collagen composite dermal hydrogel (18). Prior studies using this scaffold have demonstrated improved delivery of other cell types into wounds that have high oxidative stress (19). These technical modifications provide an improved understanding of the true effects of delivering macrophages/monocytes at supraphysiologic levels into wounds.

In conclusion, we have demonstrated that increasing the number of either macrophages or monocytes in the wound site above physiologic levels during the initial stages of wound healing can accelerate healing in both wild-type and diabetic mice. These findings hold promise for translational medicine aimed at accelerating wound healing across a broad spectrum of diseases. In diabetic patients with chronic wounds, autologous transplantation of circulating blood monocytes or macrophages derived from circulating blood monocytes may represent a viable therapeutic strategy. Derivation of macrophages from autologous banked human embryonic stem cells may also be a reality. Such a model, particularly in the case of blood monocytes, meets the criteria for an easily accessible, abundant source of autologous cells for cellular therapy that do not require in vitro manipulation prior to transplantation.

\section{Methods}

Mice. Mice were bred and maintained at the Stanford University Comparative Medicine Pavilion in accordance with Stanford University guidelines. All the animals were housed in sterile microinsulators and given water and rodent chow ad libitum. FVB/NJ, FVB-Tg(CAG-luc,-GFP)L2G85Chco/J, and FVB. $B K S(D)-L e p r^{d b} / C h u a J$ mouse strains were obtained from The Jackson Laboratory. Crl:CD1-Foxn $1^{n u}$ mice were obtained from Charles River Laboratories.

Macrophage differentiation. Mouse macrophages were generated as previously described (12). Briefly, bone marrow cells were isolated from $F V B-T g(C A G-l u c,-G F P) L 2 G 85 C h c o / J$ mice and differentiated in IMDM+- 


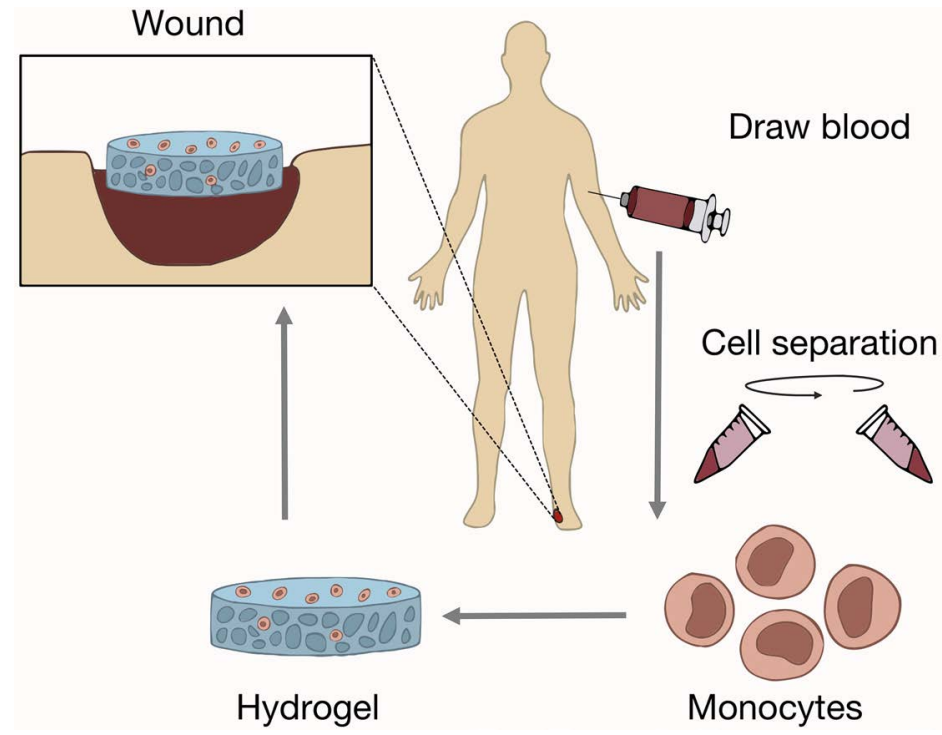

Figure 7. Schematic of autologous monocyte therapy. Autologous monocytes are first isolated from peripheral blood by density-based centrifugation or cell sorting. Monocytes are then seeded on an inert dermal hydrogel and transplanted onto extensive or chronic nonhealing wounds in the patient. This procedure would allow for same-day isolation and transplantation of autologous macrophages with no in vitro manipulation required.

GlutaMax (Life Technologies) supplemented with 10\% fetal bovine serum (HyClone), $100 \mathrm{U} / \mathrm{ml}$ penicillin, $100 \mathrm{mg} / \mathrm{ml}$ streptomycin (Life Technologies), and $10 \mathrm{ng} / \mathrm{ml}$ mouse M-CSF (Peprotech). Cells were cultured on petri dishes and incubated at $37^{\circ} \mathrm{C}$ with $5 \%$ carbon dioxide for approximately 7 days, at which point they exhibited morphological changes characteristic of macrophage development. Macrophages were prepared for transplantation by washing plates twice with PBS, incubating plates for approximately 10 minutes with TrypLE (Life Technologies) at $37^{\circ} \mathrm{C}$, and then removing cells from plates with cell lifters (Corning). Macrophages were washed and centrifuged to remove excess TrypLE and then counted and diluted to appropriate concentrations for transplantation.

Wound healing model and assessment. A splinted full-thickness excisional model of murine wound healing was utilized (10) to minimize wound contracture. Briefly, a 6-mm full-thickness excisional wound was created in duplicate on the shaved dorsum of anesthetized mice. A donut-shaped silicone splint with a $10-\mathrm{mm}$ diameter was centered on the wound and fixed to the skin using an adhesive (Krazy Glue) and interrupted 6-0 nylon sutures (Ethicon). A transparent dressing (Tegaderm) was then applied to the wound. Dressings were changed every other day beginning on day 4 , at which times gross images were taken. Wound healing was assessed by determining the area of the wound as a ratio to the inner diameter of the silicone splint using Adobe Photoshop CS6 (Adobe). Similarly, scar size was determined as a percentage of the original wound.

Macrophage transplantation. After 10 days in culture with M-CSF, adherent cells exhibited the characteristic morphology of macrophage differentiation as previously described (12). Macrophages were then seeded (in PBS) onto pullulan-collagen composite dermal hydrogels and transplanted $\left(2.5 \times 10^{5}\right.$ cells per wound) onto 6-mm full-thickness splinted excisional wounds on the dorsum of wild-type, diabetic, or immunodeficient mice at the time of wounding. At this time, control unseeded hydrogels (PBS only) were also transplanted, and wound healing outcomes were assessed. The survival, localization, and behavior of transplanted macrophages in the wound site were characterized using IVIS imaging and histologic analysis of GFP fluorescence.

Histology and tissue analysis. For fixation, tissues were placed in 2\% paraformaldehyde for $12-16$ hours at $4^{\circ} \mathrm{C}$. Samples were prepared for embedding by being soaked in $30 \%$ sucrose in PBS at $4^{\circ} \mathrm{C}$ for 24 hours. Samples were removed from the sucrose solution, and tissue blocks were prepared by embedding in Tissue Tek O.C.T. (Sakura Finetek) under dry ice to freeze the samples within the compound. Frozen blocks were mounted on a MicroM HM550 cryostat (MICROM International GmbH) and 7- to 8-micron-thick sections were transferred to Superfrost/Plus adhesive slides (Fisher Scientific).

Immunohistochemistry. For Masson's trichrome staining, standardized protocols were used without modifications. Immunostaining on frozen sections was performed using anti-CD31 (Abcam; ab28364) primary antibody. Briefly, slides were blocked for 30 minutes in 10\% BSA with 5\% horse serum, followed by incubation with primary antibody for 12-16 hours. Slides were then incubated for 1 hour with Alexa 
Fluor 647 conjugate secondary antibody (Invitrogen; A-21245). For analysis of GFP fluorescence, cryosections were incubated with DAPI at room temperature and then washed in PBS prior to mounting with Fluoromount. Fluorescent and bright-field images were taken with a Leica DM4000B microscope (Leica Microsystems) and RETIGA 2000R camera (QImaging Scientific Cameras).

Tensile strength testing. Tensile strength tests were performed on fully healed wounds on the final day that photographic images were taken, as previously described (20). Tests were performed with a microtester (Instron, model 5848; Supplemental Figure 3) equipped with a 100-N load cell. The tissue specimens were attached to custom grips with double-sided tape giving a final gauge length of $10 \mathrm{~mm}$. The region between the grips (gauge region) was stretched until a break in the skin was detected by a decrease in stress with increasing strain. Analysis was performed as previously described. True strain was calculated as the change in length divided by the gauge length, and true stress was calculated as the force divided by the original cross-sectional area. The tensile strength was determined as the greatest true stress achieved.

Quantification of scar depth. Images of macrophage- or monocyte-treated and control wounds stained for Masson's trichrome were analyzed for dermal depth ratio. Values were quantified using Adobe Photoshop CS6, and depth ratios were calculated by taking pixel measurements. The depth ratio was calculated as the average of 3 different measurements of scar depth divided by an average of the 3 different measurements of unwounded dermal depth. In this analysis, 3 sections per wound were averaged, with a total of at least 10 wounds across 5 animals for both macrophage- or monocyte-treated and control wounds.

Microfluidic single-cell gene expression analysis. Following application of macrophages to excisional cutaneous wounds, tissue was harvested from the delivery site after 24 hours operatively, minced, and digested for 1 hour at $37^{\circ} \mathrm{C}$ using collagenase I (Roche Applied Science). The reaction was stopped, and the cells were spun down and resuspended as a single-cell suspension in buffer (PBS with $2 \%$ fetal bovine serum). $\mathrm{GFP}^{+}$cells were sorted as single cells using a FACSAria flow cytometer (Becton Dickinson) into $6 \mu 1$ of lysis buffer. Cultured macrophages were sorted as a control population. Reverse transcription and low-cycle preamplification were performed using Cells Direct (Invitrogen) with TaqMan assay primer sets (Applied Biosystems) as per the manufacturer's specifications. cDNA was loaded onto 96.96 Dynamic Arrays (Fluidigm) for qPCR amplification using Universal PCR Master Mix (Applied Biosystems) with a uniquely compiled TaqMan assay primer set as previously described (13). For single-cell transcriptional data, a Kolmogorov-Smirnov test was used to compare empirical distributions, followed by an adaptive fuzzy c-means clustering algorithm, as previously described (13).

Human monocyte purification. Human peripheral blood monocytes were isolated as previously described (12). Briefly, leukocyte reduction system chambers were obtained from anonymous healthy and diabetic (determined by past medical history at time of donation; hemoglobin A1c levels unknown) blood donors at the Stanford Blood Center. The human monocytes for each experiment were obtained from a single unidentified donor of unknown sex at least 18 years of age with weight criteria based on height and excluded for HIV/AIDS, cancer (leukemia, lymphoma, multiple myeloma, and all other hematologic malignancies), heart disease, hepatitis, and organ failure (details available on the Stanford Blood Center website: https://bloodcenter.stanford.edu/donate/am-i-eligible-to-donate-blood/). Samples were incubated with $\mathrm{CD}_{1}{ }^{+}$whole-blood microbeads (Miltenyi) according to the manufacturer's instructions. CD $14^{+}$monocytes were purified by whole-blood-positive selection using an autoMACS Pro Separator (Miltenyi). Monocytes were washed with medium and then imbedded in hydrogel for transplantation.

Monocyte transplantation. Monocytes isolated by autoMACS purification were seeded (in PBS) onto pullulan-collagen composite dermal hydrogels and transplanted $\left(2.5 \times 10^{5}\right.$ cells per wound $)$ onto 6 -mm full-thickness splinted excisional wounds on the dorsum of immunodeficient $C r l: C D 1-F o x n 1^{n u}$ mice at the time of wounding. At this time, control unseeded hydrogels (PBS only) were also transplanted, and wound healing outcomes were assessed.

Statistics. GraphPad Prism version 6.0c was used for analysis of data and generation of graphs. Twogroup comparisons were analyzed using a 2-tailed unpaired Student's $t$ test. Results are expressed as mean \pm SEM. A $P$ value of less than 0.05 was considered significant.

Study approval. Animal studies were approved by the Institutional Animal Care and Use Committee Administrative Panel on Laboratory Animal Care (APLAC) at Stanford University in Stanford, California, USA (protocol APLAC-21308). 


\section{Author contributions}

MSH, GGW, KW, and MTL designed research studies. MSH, GGW, LAB, KW, RCR, DD, MJ, ZNM, WXH, ATMC, TL, CDM, RCR, SM, and ALM conducted experiments and acquired data. MSH, GGW, LAB, RCR, DD, MJ, ZNM, WXH, JR, HPL, ILW, GCG, and MTL analyzed data. HPL, ILW, GCG, and MTL provided reagents. MSH, GGW, HPL, ILW, GCG, and MTL wrote the manuscript.

\section{Acknowledgments}

The authors thank Reinhold H. Dauskardt for graciously allowing use of a microtester and Mimi Borrelli, Kelly McKenna, and Amira Barkal for their technical assistance. MSH was supported by the California Institute for Regenerative Medicine Clinical Fellow training grant (TG2-01159) and the Stanford University School of Medicine Transplant and Tissue Engineering Fellowship Award. MSH, HPL, and MTL were supported by the American Society of Maxillofacial Surgeons/Maxillofacial Surgeons Foundation Research Grant Award. HPL was supported by NIH grant R01 GM087609 and a gift from Ingrid Lai and Bill Shu in honor of Anthony Shu. HPL, GCG, and MTL were supported by the Hagey Laboratory for Pediatric Regenerative Medicine and The Oak Foundation. ILW was supported by the Ludwig Foundation. ILW and MTL were supported by the Gunn/Olivier Fund. MTL was supported by NIH grant U01 HL099776.

Address correspondence to: Geoffrey C. Gurtner or Michael T. Longaker, Hagey Laboratory for Pediatric Regenerative Medicine, Stanford University School of Medicine, 257 Campus Drive, Stanford, California 94305-5461, USA. Phone: 650.736.2776; Email: ggurtner@stanford.edu (G.C. Gurtner). Phone: 650.736.1707; Email: longaker@stanford.edu (M.T. Longaker).

1. Sen CK, et al. Human skin wounds: a major and snowballing threat to public health and the economy. Wound Repair Regen. 2009;17(6):763-771

2. Werner S, Grose R. Regulation of wound healing by growth factors and cytokines. Physiol Rev. 2003;83(3):835-870.

3. Daley JM, Brancato SK, Thomay AA, Reichner JS, Albina JE. The phenotype of murine wound macrophages. JLeukoc Biol. 2010;87(1):59-67.

4. Fang Y, Gong SJ, Xu YH, Hambly BD, Bao S. Impaired cutaneous wound healing in granulocyte/macrophage colony-stimulating factor knockout mice. Br J Dermatol. 2007;157(3):458-465.

5. Leibovich SJ, Ross R. The role of the macrophage in wound repair. A study with hydrocortisone and antimacrophage serum. Am J Pathol. 1975;78(1):71-100.

6. Mirza R, DiPietro LA, Koh TJ. Selective and specific macrophage ablation is detrimental to wound healing in mice. $A m J$ Pathol. 2009;175(6):2454-2462.

7. Lucas T, et al. Differential roles of macrophages in diverse phases of skin repair. J Immunol. 2010;184(7):3964-3977.

8. Gu XY, et al. Effect of activated autologous monocytes/macrophages on wound healing in a rodent model of experimental diabetes. Diabetes Res Clin Pract. 2013;102(1):53-59.

9. Jetten N, et al. Wound administration of M2-polarized macrophages does not improve murine cutaneous healing responses. PLoS One. 2014;9(7):e102994.

10. Galiano RD, Michaels J, Dobryansky M, Levine JP, Gurtner GC. Quantitative and reproducible murine model of excisional wound healing. Wound Repair Regen. 2004;12(4):485-492.

11. Cao YA, et al. Shifting foci of hematopoiesis during reconstitution from single stem cells. Proc Natl Acad Sci U S A. 2004;101(1):221-226.

12. Weiskopf $\mathrm{K}$, et al. Engineered $\operatorname{SIRP} \alpha$ variants as immunotherapeutic adjuvants to anticancer antibodies. Science. 2013;341(6141):88-91.

13. Glotzbach JP, et al. An information theoretic, microfluidic-based single-cell analysis permits identification of subpopulations among putatively homogeneous stem cells. PLoS One. 2011;6(6):e21211.

14. Chua S, Liu SM, Li Q, Yang L, Thassanapaff VT, Fisher P. Differential $\beta$ cell responses to hyperglycaemia and insulin resistance in two novel congenic strains of diabetes (FVB- Lepr (db)) and obese (DBA- Lep (ob)) mice. Diabetologia. 2002;45(7):976-990

15. Santoro MM, Gaudino G. Cellular and molecular facets of keratinocyte reepithelization during wound healing. Exp Cell Res. 2005;304(1):274-286.

16. Gordon S. Alternative activation of macrophages. Nat Rev Immunol. 2003;3(1):23-35.

17. Hamilton JA. Colony-stimulating factors in inflammation and autoimmunity. Nat Rev Immunol. 2008;8(7):533-544.

18. Wong VW, et al. Engineered pullulan-collagen composite dermal hydrogels improve early cutaneous wound healing. Tissue Eng Part A. 2011;17(5-6):631-644.

19. Wong VW, et al. Pullulan hydrogels improve mesenchymal stem cell delivery into high-oxidative-stress wounds. Macromol Biosci. 2011;11(11):1458-1466.

20. Rinkevich Y, et al. Skin fibrosis. Identification and isolation of a dermal lineage with intrinsic fibrogenic potential. Science. 2015;348(6232):aaa2151. 